\title{
The Free Base Extraction of Harmaline from Penganum harmala
}

\author{
Alyssa Brobst, Jeremy Lewis, Brian Klett, Cathy Haustein, \\ and James Shriver \\ Department of Chemistry \\ Central College \\ Pella, lowa 50219 USA
}

Received: May 11, 2009 Accepted: November 30, 2009

\begin{abstract}
Highly fluorescent and pharmaceutically significant Harmala alkaloids occur in several plants including Syrian Rue (Penganum harmala) and several species of passion flowers. We identified a relatively nontoxic and efficient method for extraction of the major harmala alkaloid components from Syrian Rue seeds using ethyl acetate and sodium bicarbonate. This produced free base harmala alkaloids. Verification was performed qualitatively by using High Performance Liquid Chromatography and NMR analysis.
\end{abstract}

\section{INTRODUCTION}

According to records kept by explorers in the early 1800 s, Syrian Rue (Penganum harmala) has long been used by the indigenous peoples of Central America for its psychological effects. The plant is also natively found in Central Asia and Syria. Syrian Rue was first used by native peoples for its pharmaceutical value and as a dye. The natives used the plant primarily for its psychoactive effects. However, when ingested alone, it can take up to two hours for the psychological effects of the active component, harmaline, to be apparent [1].

The medical value of harmala alkaloids, the active chemicals in the Syrian Rue plant, has been debated. The most significant harmala alkaloid in Syrian Rue is harmaline. This extract has been of interest primarily for its use as a mild sleep-inducer. It has been postulated that harmala alkaloids have pharmaceutical significance as potential sleep inducers, especially in pediatric and geriatric applications. Though the Syrian Rue plant has not been heavily studied for its therapeutic value, Passiflora plants have. Harmala alkaloids are present in Passiflora at less than 0.01 percent and are not soluble in water. Nonetheless, it has been found that the constituents of
Passiflora with sedative effects are water soluble. Finally, researchers have found harmala alkaloids to have stimulant rather than sedative effects [3].

Harmaline has also been utilized in the medical research field for its tremorgenerating effects. When isolated, this chemical can be used to study the mechanisms of tremorgenesis by way of animal models, specifically rats. It must be noted that harmaline-induced tremors mimic essential tremors, but not those characteristic of Parkinson's disease. Researchers not only hope to better understand tremor mechanisms through this process, but will use the information acquired from such studies to develop respective treatments [2].

\section{MATERIALS}

A Hewlett Packard 1100 Series HPLC with a Phenomenex c-18 Luna column $250 \mathrm{~mm}$ by $4.60 \mathrm{~mm}$ : UV-VIS detection at $340 \mathrm{~nm}, 20 \mu \mathrm{L}$ injection loop was used for the chromatographic analysis. The flow rate was set at $1 \mathrm{ml} /$ minute. The column was heated to $30^{\circ} \mathrm{C}$ with a phenomenex ThermaSphere column heater. A gradient of DI water and methanol was used to help increase the resolution of harmine and 
harmane. The mixture was $25 \%$ DI water and $75 \%$ pure methanol for 2 minutes, followed by $100 \%$ methanol for the remaining 8 minutes. Brüker $300 \mathrm{MHz} \mathrm{NMR}$ was used for spectroscopic measurement; the solvent used for analysis was deuterated chloroform. Reagents were obtained from Sigma-Aldrich and used as received. Syrian Rue seeds were purchased from Gaia's Delight.

Procedure

Three different extraction procedures were used in order to obtain harmala alkaloids from Syrian Rue seeds. The three methods of extraction involve both the acidification of the alkaloids, followed by the removal of impurities with organic solvent, and basification of the solution followed by the extraction of the alkaloids with the organic solvent. The first extraction involved the use of chloroform and sodium hydroxide, the second ethyl acetate and sodium bicarbonate, and the third ethyl acetate and sodium hydroxide. The extraction method was based on a previously developed method [4].

For each extraction, approximately $10 \mathrm{~g}$ of Syrian Rue seeds were used. The seeds were ground to a fine powder using a coffee grinder and placed in a clean, dry beaker. To the beaker was added five times the weight of the seeds in milliliters (approximately $50 \mathrm{~mL}$ ) of an acetic acid solution. The solution contained $30 \mathrm{~g}$ of acetic acid per liter of water. The mixture was placed on a stir plate for five minutes at a low speed. Following the five minutes, the solid material was removed using a filter system with a Bucher funnel lined with Whatman 4 filter paper. During the filtering process, the plant material was washed twice with the aforementioned acetic acid solution (each wash was approximately $10 \mathrm{~mL})$.

After the plant material was filtered, $50 \mathrm{~mL}$ of petroleum ether and $50 \mathrm{~mL}$ of ethyl acetate were added to a separatory funnel along with the resulting solution from the plant material and acetic acid solution in order to remove any organic impurities. The solution was gently mixed by way of inversion and the aqueous layer was removed for later use. Leaving the top layer in the separatory funnel, and addition $50 \mathrm{~mL}$ of petroleum ether and $50 \mathrm{~mL}$ of ethyl acetate was added, mixed, and again the aqueous layer was removed. This process was repeated once more for a total of three times.

To the saved aqueous solution, an aqueous base was added drop wise until the solution turned basic. After every five to ten drops had been added, the solution was stirred and the basicity was tested using $\mathrm{pH}$ paper. When the transformation had occurred, the $\mathrm{pH}$ paper turned blue in color and the solution went from being a transparent brown color to a cloudy, light brown color.

Finally, $100 \mathrm{~mL}$ of the organic solvent was added to the separatory funnel along with the now basic solution. The contents of the funnel were mixed gently by inversion and the layer containing the organic solvent was collected. To the solution still in the funnel, an additional $100 \mathrm{~mL}$ of the solvent was added and the organic solvent layer was again collected. This was repeated one more time, for a total of three total extractions. After the final collection, sodium sulfate was added to the solution in order to remove any excess water. (It is not important to add a specified amount of sodium sulfate to the solution; when the sodium sulfate ceases to clump, this is an indication that the water has been removed.) The now dry solution was poured through a glass funnel, using cotton as a filter, into a preweighed $500 \mathrm{~mL}$ round bottom flask. The cotton ball was washed with approximately $5 \mathrm{~mL}$ of the organic solvent to ensure that all of the extract was contained in the round bottom flask. After the filtration, the solvent was removed from the solution using rotary evaporation using an aspirator. For further drying, a low pressure pump was used to pump out any remaining solvent. After the pumping process was finished, the flask was weighed to obtain a yield.

For the first extraction, the organic solvent used was chloroform and the aqueous base was $1 \mathrm{M} \mathrm{NaOH}$. In this procedure, $10.0768 \mathrm{~g}$ of Syrian Rue seeds were used. The second extraction involved the organic solvent ethyl acetate and the aqueous base sodium bicarbonate. In this procedure, $10.0743 \mathrm{~g}$ of Syrian Rue seeds were used. In the third and final extraction, the organic solvent used was ethyl acetate and the aqueous base was $1 \mathrm{M} \mathrm{NaOH}$. In this procedure, $9.9929 \mathrm{~g}$ of Syrian Rue seeds were used. 


\begin{tabular}{|l|c|c|c|c|c|}
\hline \multicolumn{1}{|c|}{ Extraction } & $\begin{array}{c}\text { HPLC } \\
\text { (Harmaline } \\
\text { Area/ Grams } \\
\text { of seeds) } \\
\text { (Average of 2 } \\
\text { Trials, 2009) }\end{array}$ & $\begin{array}{c}\text { NMR } \\
\text { (Harmaline: } \\
\text { Harmine } \\
\text { Mole Ratio) } \\
\text { (2009) }\end{array}$ & $\begin{array}{c}\text { NMR } \\
\text { (Harmaline: } \\
\text { Harmine } \\
\text { Mole Ratio) } \\
\text { (2005-2006) }\end{array}$ & $\begin{array}{c}\text { Percent } \\
\text { Recovery } \\
\text { (1 Trial, } \\
\text { 2009) }\end{array}$ & $\begin{array}{c}\text { Percent } \\
\text { Recover } \\
\text { (Average } \\
\text { of 3 Trials, } \\
\text { 2005-2006) }\end{array}$ \\
\hline $\begin{array}{l}\text { Chloroform and } \\
\text { NaOH }\end{array}$ & 89.1 & $1.0: 1.1$ & $1.0: 0.8$ & $2.50 \%$ & $1.38 \%$ \\
\hline $\begin{array}{l}\text { Ethyl Acetate and } \\
\text { Sodium Bicarbonate }\end{array}$ & 127.2 & $1.0: 0.8$ & $1.0: 1.0$ & $2.95 \%$ & $0.97 \%$ \\
\hline $\begin{array}{l}\text { Ethyl Acetate and } \\
\text { NaOH }\end{array}$ & 125.5 & $1.0: 1.9$ & - & $2.91 \%$ & - \\
\hline
\end{tabular}

Table 1. Comparison of Extraction Methods: The NMR peaks present from the three extractions are as follows: Harmaline [ $\delta(\mathrm{ppm})]: 7.482(\mathrm{~d}, \mathrm{~J}-8.7 \mathrm{~Hz}), 6.871(\mathrm{~d}, \mathrm{~J}=1.8 \mathrm{~Hz}), 6.842$ (dd, J=8.7Hz and $2.1 \mathrm{~Hz}), 3.872(\mathrm{~s}), 3.843(\mathrm{t}, \mathrm{J}=8.7 \mathrm{~Hz}), 2.845(\mathrm{t}, \mathrm{J}=8.7 \mathrm{~Hz})$, and $2.378(\mathrm{~s})$. Harmine $[\delta(\mathrm{ppm})]$ : $8.339(\mathrm{~d}, \mathrm{~J}=5.4 \mathrm{~Hz}), 7.986(\mathrm{~d}, \mathrm{~J}=8.7 \mathrm{~Hz}), 7.736(\mathrm{~d}, \mathrm{~J}=5.4 \mathrm{~Hz}), 6.992(\mathrm{~d}, \mathrm{~J}=2.1 \mathrm{~Hz}), 6,925$ (dd, $\mathrm{J}=8.6 \mathrm{~Hz}$ and $2.3 \mathrm{~Hz}), 3.935(\mathrm{~s})$, and $2.816(\mathrm{~s})$.

After the extraction processes, an NMR and HPLC was taken for each extract. The HPLC standards used were harmaline and harmine. In doing the HPLC, $0.0125 \mathrm{~g}$ of the solid (of both the standards and the extracts) was dissolved in methanol and diluted to $100 \mathrm{~mL}$ using a volumetric flask. The NMR was done using a qualitative amount of solid from the extract dissolved in deuterated chloroform. This procedure was performed once during the years of 2005-2006 and again in 2009.

\section{RESULTS}

The yields obtained are as follows: $0.2507 \mathrm{~g}$ of precipitate was obtained from the chloroform and $\mathrm{NaOH}$ extraction, $0.2972 \mathrm{~g}$ of precipitate was obtained from the ethyl acetate and sodium bicarbonate extraction, and $0.2909 \mathrm{~g}$ of precipitate was obtained from the ethyl acetate and $\mathrm{NaOH}$ extraction. These values are those given for the experiment performed in 2009. Table 1 also summarizes the information obtained in 2005-2006. Upon HPLC analysis, harmaline was identified by the retention time of 5.75.8 , which was verified by the standard addition to each extract. Harmine was not seen in any of the HPLC analyses. NMR analysis displayed the mole ratio of harmine to harmaline (Table 1).

\section{CONCLUSIONS}

As seen in Table 1, both the traditional method for harmala alkaloid extractions using chloroform and $\mathrm{NaOH}$ and the new methods using ethyl acetate and sodium bicarbonate and ethyl acetate and $\mathrm{NaOH}$ extracted the same harmalas. The HPLC indicated the extraction of harmaline but not harmine, while the NMR analysis was more sensitive and indicated the presence of both harmaline and harmine (Table 1). In addition, the three extraction methods relatively equal amounts of solid, as seen in the percent recovery values (Table 1).

This data indicates that the safest of the three extraction methods, involving ethyl acetate and sodium bicarbonate, could be used a substitute for the traditional chloroform and $\mathrm{NaOH}$ extraction. In addition, the research done on harmala alkaloids indicate that harmaline is the most useful of those present in Syrian Rue (CITATION). Though the HPLC indicated 
nearly equal amounts of harmaline present after the three different extractions (Table 1), the NMR showed less similar results. Table 1 indicates that the proposed new method of extraction would be best for extracting this chemical as it produces the lowest harmine to harmaline mole ratio. Further purification to remove any harmine would be necessary.

\section{ACKNOWLEDGEMENTS}

We thank the Monticello Foundation for providing the grant necessary to fund the research performed by Alyssa Brobst and Cathy Haustein.

\section{REFERENCES}

1. Stafford, Peter. Ayahuasca, Yagé and Harmaline. Psychedelics Encyclopedia. (Ronin Publishing, 1993).

Accessed 15 April 2009:

http://www.biopark.org/peru/Ayaharmali ne.html>.
2. Miwa, Hideto. Rodent models of tremor. Cerebellum 6(1) (March 2007), pp. 6672. Academic Search Premier. EBSCO. Geisler Library, Pella, IA.

Accessed 29 April 2009:

http://proxy.central.edu:2272/login.aspx

?direct=true $\& \mathrm{db}=\mathrm{aph} \& \mathrm{AN}=24325431 \& \mathrm{si}$ te=ehost-live.

3. Bergner, Paul. Passiflora: Passion flower. Medical Herbalism (2001).

Accessed 15 April 2009: http:// medherb.com/Materia_Medica/Passiflor a_-_Passion flower_.htm.

4. Frye, Abigail, and Catherine Haustein. Extraction, Identification, and Quantification of Harmala Alkaloids in Three Species of Passiflora. American Journal of Undergraduate Research 6(3) (2007), pp. 19-26.

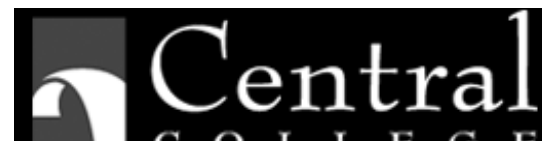

ga farther than you ever dreamed!

Study Chemistry at Pella College (Pella, lowa)

Individualized attention, great research opportunities and in-depth instruction - these are the hallmarks of the Central College chemistry department. Chemistry majors at Central take the same courses as students at larger institutions but have greater opportunities for research and mentoring due to the one-on-one attention provided by the Central faculty. The chemistry department is certified by the prestigious American Chemical Society which means graduates are ensured to have a strong scientific background needed for graduate school or careers in chemistry. http://www.central.edu/academics/majors/chemistry/ 